Research Article

\title{
Time-Delayed Polynomial Grey System Model with the Fractional Order Accumulation
}

\author{
Lin Chen (D), Zhibin Liu, and Nannan Ma \\ School of Science, Southwest Petroleum University, Chengdu, Sichuan, China \\ Correspondence should be addressed to Lin Chen; chenlin8976@163.com
}

Received 25 September 2018; Accepted 13 November 2018; Published 25 November 2018

Academic Editor: Raquel Caballero-Águila

Copyright (C) 2018 Lin Chen et al. This is an open access article distributed under the Creative Commons Attribution License, which permits unrestricted use, distribution, and reproduction in any medium, provided the original work is properly cited.

In this work, a novel time-delayed polynomial grey prediction model with the fractional order accumulation is put forward, which is abbreviated as TDPFOGM(1,1), based on the new grey system theory to predict the small sample in comparison with the existing forecasting models. The new model takes into account the nonhomogeneous term and the priority of new information can be better reflected in the in-sample model. The data in this paper all come from the existing literatures. The results demonstrate that the TDPFOGM(1,1) model outperforms the TDPGM(1,1) and FOGM(1,1) model.

\section{Introduction}

Shale gas is one kind of fossil fuel which is clean, efficient, and cheap. With the shale gas revolution in the United States $[1,2]$, shale gas has been developed in many countries. And China has achieved some success [3]. The development of shale gas industry has been regarded as an important mission of the government $[4,5]$. In such circumstances, it is very important to predict the natural gas production and consumption in China for the decision-makers, such as the government and energy companies.

Grey model proposed by Deng [6] is used for small sample forecasting. The grey prediction models play an important role in the grey system theory. These models are often named as grey models. $\operatorname{GM}(1,1)$, that is, first-order Grey model with one variable, has been successfully applied in many disciplines [7-13]. However, the existing $\operatorname{GM}(1,1)$ model cannot be accurate prediction for many actual systems. In recent literatures [14-23], the improved grey prediction models are popularly used for energy consumption. Ma and Liu [14] have proposed a novel time-delayed polynomial grey model to predict the natural gas consumption in China. Akay and Atak [15] have proposed a novel method based on the basic $\operatorname{GM}(1,1)$ model with rolling mechanism to forecast the electricity demand of Turkey. Wu and Shen [16] have proposed a grey-related least squares support vector machine optimization model and applied it, predicting the natural gas consumption demand. The authors in [17] have proposed a novel kernel regularized nonhomogeneous grey model and applied it, forecasting the petroleum production. Kumar and Jain [18] have used the Grey-Markov and $\operatorname{GM}(1,1)$ model with rolling mechanism to predict the energy consumption in India. In [19], a prediction method using grey model for cumulative plastic deformation under cyclic loads was demonstrated. Pao and Tsai [22] have used the GM(1,1) model to predict the energy consumption in Brazil compared with the ARIMA model.

Discrete grey model also has good effect on prediction. Ayvaz and Kusakci [24] have used the nonhomogeneous discrete grey model to predict the electricity consumption forecasting for Turkey. Xie and Pearman [25] have used the discrete $\operatorname{GM}(1,1)$ model $(\operatorname{DGM}(1,1))$ to predict the energy consumption of China. Simultaneously, the fractional gray model is proposed. In [26], the authors have proposed a grey system model with the fractional order accumulation. Yuan et al. [27] have pointed out the advantages and disadvantages of GM $(1,1)$ and the Autoregressive Integrated Moving Average (ARIMA) model and then proposed a novel hybrid model based on the GM(1,1) model and the ARIMA model to predict primary energy consumption in China. Wu et al. [28] have proposed a novel GM $(1,1)$ model with the Principle of New Information Priority $(\operatorname{NIGM}(1,1))$ to predict the natural gas consumption in China, and the results show that $\operatorname{NIGM}(1,1)$ 
outperforms several existing models. Mao et al. [29] present a new fractional grey model, in which first-order differential equations are transformed into fractional differential equations. And it has high modeling precision and can overcome the $\operatorname{GM}(1,1)$ model class ratio test restrictions. Yang et al. [30] modified optimized fractional grey model using the error feedback, and the performance is evaluated and greatly improved in modeling. Meng et al. [31] develop a discrete grey model with fractional operators, which also makes use of genetic algorithms to optimize the modeling parameter. Yang et al. [32] proposed that the geometric coordinate features are used by the coordinates of area and middle point lines and established the grey prediction model for interval grey number by the fractional order accumulation calculus. Wu et al. [33] developed a novel multivariable grey forecasting model that considered the total population to forecast the electricity consumption. Li et al. [34] proposed a novel grey forecasting model with full-order time power terms (FOTP-GM(1,1)). Wang et al. [35] propose a data grouping approach based grey modeling method DGGM $(1,1)$ to predict quarterly hydropower production in China. All these researches indicate that the grey models are efficient to predict the consumption of many kinds of energy for many countries.

Motivated by the above, in this paper, we propose a novel time-delayed polynomial grey model with the fractional order accumulation. Simultaneously, the model considered the character time-delayed polynomial and fractional order. The main contributions of this paper are summarized as follows: (1) this paper is based on predecessor's research about grey model; a new model is put forward; (2) using the same data, the TDPFOGM(1,1) model makes predictions just as shown in figures. And the results were compared with the $\operatorname{TDPGM}(1,1)$ model and the FOGM(1,1) model; (3) more intuitively, the mean absolute percentage error (MAPE) is shown in the tables.

The rest of this paper is organized as follows: the details of modeling procedures of the TDPFOGM $(1,1)$ model are given in Section 2; the application of $\operatorname{TDPFOGM}(1,1)$ to small sample prediction is given in Section 3, including the comparison with the other commonly used prediction models presented based on the same data sample, and the conclusions are drawn in Section 4.

\section{Description of the Problem}

In this section, we will present the time-delayed polynomial grey system model with the fractional order accumulation, abbreviated as the $\operatorname{TDPFOGM}(1,1)$ or $\operatorname{TDPGM}^{(p / q)}(1,1)$ model, including the principles and the computational steps.

2.1. Grey Model with the Fractional Order Accumulation. Let the $r$ th $\left(r \in R_{+}\right)$order accumulated generating operator of the original nonnegative sequence $X^{(0)}$ be $X^{(r)}, r=1,2, \ldots, n$.

$$
\begin{aligned}
& \text { Set }\left(\begin{array}{c}
p / q-1 \\
0
\end{array}\right)=1 ;\left(\begin{array}{c}
k-1 \\
k
\end{array}\right)=0, k=1,2, \ldots, n \text {; then } \\
& X^{(r)}(k)=\sum_{i=1}^{k}\left(\begin{array}{c}
k-i+r-1 \\
k-i
\end{array}\right) x^{(0)}(i), \quad k=1,2, \ldots, n
\end{aligned}
$$

where $\left(\begin{array}{c}k-i+r-1 \\ k-i\end{array}\right)=(r+k-i-1)(r+k-i-2) \cdots(r+1) r /$ $(k-i)$ !

Fractional derivatives accumulate the whole history of the system in weighted form. $x^{(1)}(k)$ in grey system theory denotes the weight of $x^{(0)}(i)(i=1,2, \ldots, k)$ as 1 . The larger $r$ of $x^{(r)}(k)$ is the larger the weight of old data is; the smaller $r$ of $x^{(r)}(k)$ is the smaller the weight of old data is. Reducing $r$ can reduce the weights of old data, which can put more emphasis on the newer data (more details and the properties of the fractional order accumulation generation operation can be seen in [25]).

The original form of the $\operatorname{GM}^{p / q}(1,1)$ model is as follows:

$$
x^{(p / q)}(k)-x^{(p / q)}(k-1)+a z^{(p / q)}(k)=b
$$

If $r=p / q$, then $(p / q)(0<p / q \leq 1)$ order inverse accumulated generating operator of $X^{(0)}$; we write

$$
\begin{gathered}
\alpha^{(p / q)} X^{(0)}=\alpha^{(1)} X^{(1-p / q)}(k)=\left\{\alpha^{(1)} x^{(1-p / q)}(1),\right. \\
\left.\alpha^{(1)} x^{(1-p / q)}(2), \ldots, \alpha^{(1)} x^{(1-p / q)}(n)\right\}
\end{gathered}
$$

2.2. The Representation of the TDPFOGM(1,1) Model. The new form of the TDPFOGM $(1,1)$ model is as follows:

$$
\begin{aligned}
& x^{(p / q)}(k)-x^{(p / q)}(k-1)+\lambda_{1} z^{(p / q)}(k) \\
& =\lambda_{2} \sum_{\tau=1}^{k} \tau^{2}+\lambda_{3} \sum_{\tau=1}^{k} \tau+\lambda_{4} \\
& z^{(p / q)}(k)=\frac{x^{(p / q)}(k)+x^{(p / q)}(k+1)}{2}, \\
& k=1,2, \ldots, n-1
\end{aligned}
$$

where $z^{(p / q)}(k)$ is called the background value. For $\operatorname{TDPFOGM}(1,1)$ model, the first number "1" stands for "first order" and the second number " 1 " means "one sequence," as only one sequence is considered in this model. It is the traditional $\operatorname{TDPGM}(1,1)$ model [14], when $p / q=1$. The ordinary least squares estimate sequence of the TDPFOGM $(1,1)$ model is satisfied.

2.3. The Solution of the TDPFOGM(1,1) Model. $X^{(0)}=$ $\left\{x^{(0)}(1), x^{(0)}(2), \ldots, x^{(0)}(n)\right\}$ is the given original sequence, and the unconstrained optimization equation (6) can describe the least squares criteria for the $\operatorname{TDPFOGM}(1,1)$ model:

$$
\begin{aligned}
& \min _{\lambda_{1}, \lambda_{2}, \lambda_{3}, \lambda_{4}} \sum_{k=2}^{n}\left[x^{(p / q)}(k)-x^{(p / q)}(k-1)+\lambda_{1} z^{(p / q)}(k)\right. \\
& \left.-\lambda_{2} \sum_{\tau=1}^{k} \tau^{2}-\lambda_{3} \sum_{\tau=1}^{k} \tau-\lambda_{4}\right]^{2}
\end{aligned}
$$

The linear system in (7) can solve the above optimization problem:

$$
\left[\lambda_{1}, \lambda_{2}, \lambda_{3}, \lambda_{4}\right]^{T}=\left(B^{T} B\right)^{-1} B^{T} Y
$$


where

$$
\begin{aligned}
& B=\left[\begin{array}{cccc}
-z^{(p / q)}(2) & \frac{5}{2} & \frac{3}{2} & 1 \\
-z^{(p / q)}(3) & 9 & 4 & 1 \\
\vdots & \vdots & \vdots & \vdots \\
-z^{(p / q)}(n) & \sum_{\tau=2}^{n} \frac{n^{2}+(n-1)^{2}}{2} & \sum_{\tau=2}^{n} \frac{2 n-1}{2} & 1
\end{array}\right], \\
& Y=\left[\begin{array}{c}
x^{(p / q)}(2)-x^{(p / q)}(1) \\
x^{(p / q)}(3)-x^{(p / q)}(2) \\
\vdots \\
x^{(p / q)}(n)-x^{(p / q)}(n-1)
\end{array}\right]
\end{aligned}
$$

The equation

$$
\frac{d x^{(p / q)}(t)}{d t}+\lambda_{1} x^{(p / q)}(t)=\lambda_{2} \sum_{\tau=1}^{t} \tau^{2}+\lambda_{3} \sum_{\tau=1}^{t} \tau+\lambda_{4}
$$

is called a whitenization differential equation of TDP$\operatorname{FOGM}(1,1)$, and the solution of the whitenization equation is given by the following equation:

$$
\begin{aligned}
\widehat{x}^{(p / q)}(k) & \\
= & x^{(p / q)}(1) e^{-a(k-1)} \\
& +\sum_{\tau=2}^{k} \frac{1}{2}\left[e^{-a(k-\tau)} f(\tau)+e^{-a(k-\tau+1)} f(\tau-1)\right]
\end{aligned}
$$

where

$$
f(t)=\lambda_{2} \sum_{\tau=1}^{t} \tau^{2}+\lambda_{3} \sum_{\tau=1}^{t} \tau+\lambda_{4}
$$

Equation (10) is called the discrete response function, and within the initial condition $x^{(p / q)}(1)=x^{(0)}(1)$, the discrete response function can be obtained as follows:

$$
\begin{aligned}
\widehat{x}^{(p / q)}(k) & \\
= & x^{(0)}(1) e^{-\lambda_{1}(k-1)} \\
& +\sum_{t=2}^{k} \frac{1}{2}\left[e^{-\lambda_{1}(k-t)} f(t)+e^{-\lambda_{1}(k-t+1)} f(t-1)\right]
\end{aligned}
$$

The values of the series $x^{(p / q)}(k)$ can be computed using the response function (12), and the predicted values of the original series $x^{(0)}(k)$ can be obtained using the fractional order inverse accumulative generation operation as follows:

$$
\begin{gathered}
\widehat{X}^{(0)}=\widehat{X}^{(p / q)(-p / q)}(k)=\left\{x^{(p / q)(-p / q)}(1),\right. \\
\left.x^{(p / q)(-p / q)}(2), \ldots, x^{(p / q)(-p / q)}(n)\right\}
\end{gathered}
$$

The derivation of the parameter estimation of $\operatorname{TDPFOGM}(1,1)$ is similar to the other first order grey prediction models, such as TDPGM(1,1) (see [14]).
Remark 1. The TDPFOGM $(1,1)$ model has the properties of fractional order accumulation and the polynomial function, and it is not applicable for the periodic series and the random series and so forth according to its mathematical formulation. Exponential function and a discrete integral with an exponential and the polynomial function combination explain the discrete response function of the TDPFOGM $(1,1)$ model. The priority of new information can be better reflected when the accumulation order number becomes smaller in the model.

2.4. The Computational Steps. According to the principles of time-delayed polynomial grey system model with the fractional order accumulation, the computational steps can be summarized as follows.

Step 1. Within a given original sequence, compute the series of the given time series using (1) and compute the background values using (5).

Step 2. Substitute the original sequence along with its series and the background values into (7) and compute the parameters $\lambda_{1}, \lambda_{2}, \lambda_{3}, \lambda_{4}$ by solving the linear system in (7).

Step 3. Substitute the parameters $\lambda_{1}, \lambda_{2}, \lambda_{3}, \lambda_{4}$ into the discrete response function in (12) and then compute the series $x^{(p / q)}(k)$.

Step 4. Compute the predicted values of $x^{(0)}(k)$ using the (13).

\section{Model Application and Comparison}

3.1. Raw Data Collection. The raw data of the natural gas consumption $\left(10^{9} \mathrm{~m}^{3}\right)$ of China are collected from [14] (page 20) as shown in Table 1 . We build the prediction models using the data from 1995 to 2004 and validate the modeling accuracy using the data from 2005 to 2013.

We consider an example from paper [36]. The same sample is applied here to compare the precision. Actual values are presented in Table 2.

3.2. Evaluation Indices of the Modeling Accuracy. The mean absolute percentage error (MAPE) is used to evaluate the overall forecast performance of the prediction models, which is defined as follows:

$$
\operatorname{MAPE}=\frac{1}{n} \sum_{k=1}^{n}\left|\frac{x^{(0)}(k)-\widehat{x}^{(0)}(k)}{x^{(0)}(k)}\right| \times 100(\%)
$$

\subsection{Results and Analysis}

Case 1. We consider the data from Table 1. The same sample is applied here to compare the precision. Actual values and the fitting values of four compared models are presented in Table 3 and the predicted values are also plotted in Figure 1. From Table 3, $\operatorname{TDPGM}^{(0.25)}(1,1)$ yielded lower MAPE compared with the traditional TDPGM(1,1), GM(1,1), and $\mathrm{GM}^{(0.25)}(1,1)$. 
TABLE 1: The raw data of the natural gas consumption of China.

\begin{tabular}{lc}
\hline Year & Natural gas consumption \\
\hline 1995 & 177.53 \\
1996 & 188.05 \\
1997 & 176.13 \\
1998 & 218.7 \\
1999 & 215.34 \\
2000 & 245.03 \\
2001 & 274.3 \\
2002 & 291.84 \\
2003 & 339.08 \\
2004 & 396.72 \\
2005 & 467.63 \\
2006 & 561.41 \\
2007 & 705.23 \\
2008 & 812.94 \\
2009 & 895.2 \\
2010 & 1069.41 \\
2011 & 1305.3 \\
2012 & 1463 \\
2013 & 1705.37 \\
\hline
\end{tabular}

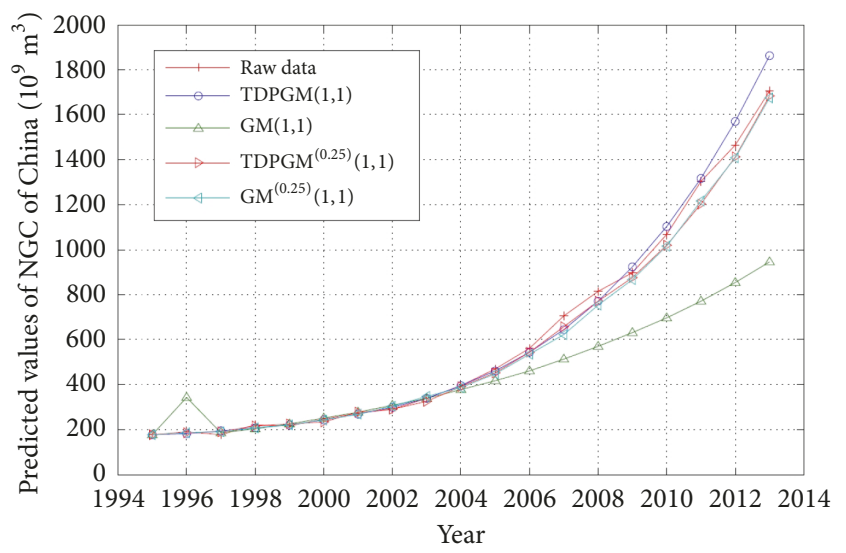

Figure 1: Prediction results by TDPGM(1,1), GM(1,1), $\operatorname{GM}^{(0.25)}(1,1)$, and $\operatorname{TDPGM}^{(0.25)}(1,1)$.

Case 2. We consider the data from Table 2. The same sample is applied here to compare the precision. Actual values and the fitting values of four compared models are presented in Table 4 and the predicted values are also plotted in Figure 2. From Table 4, $\operatorname{TDPGM}^{(0.5)}(1,1)$ yielded lower MAPE compared with the traditional $\mathrm{GM}(1,1)$ and $\mathrm{GM}^{(0.5)}(1,1)$.

Remark 2. In this paper, we established the novel grey prediction model that considers the fractional order accumulation while considering the polynomial based on the $\operatorname{TDPGM}(1,1)$ model which is different from the existing literature [14]. There are three main reasons; firstly, in [14], the authors proposed a time-delay polynomial grey model, but fractional order accumulation is not mentioned. Secondly, The TDPFOGM $(1,1)$ model is a further study on the basis of

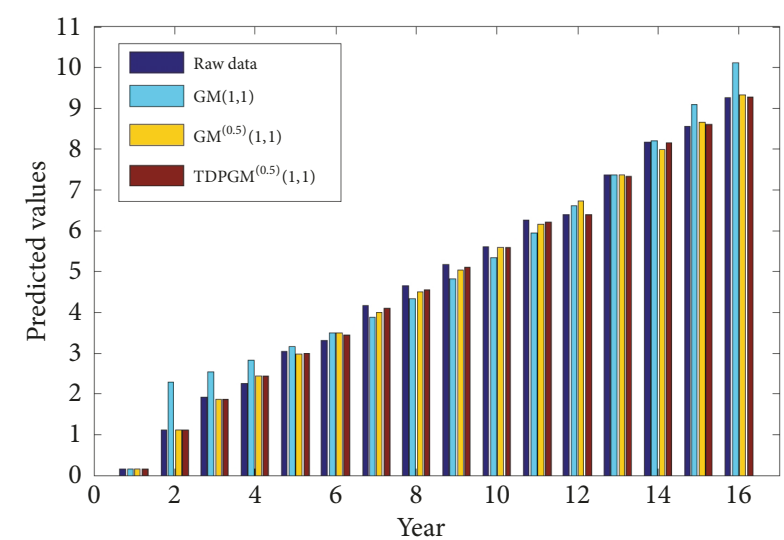

FIGURE 2: Prediction results by $\operatorname{GM}(1,1), \operatorname{GM}^{(0.5)}(1,1)$, and $\operatorname{TDPGM}^{(0.5)}(1,1)$.

the TDPGM $(1,1)$ model and TDPGM $(1,1)$ model is a special case when fractional order $p / q=1$. Finally, The prediction performance of TDPFOGM $(1,1)$ model is better than that of the $\operatorname{TDPGM}(1,1)$ model according to the numerical results and analysis by comparing the TDPFOGM $(1,1)$ and $\operatorname{TDPGM}(1,1)$ models based on the same data sample.

\section{Conclusion}

Small sample forecasting is a difficult and important problem. We have improved the grey prediction model after studying the grey system model with the fractional order accumulation and the time-delayed polynomial grey model. Based on a series of analysis and derivation for grey prediction system, the following conclusions could be drawn:

(1) Time-delayed polynomial grey system model with the fractional order accumulation (TDPFOGM $(1,1)$ ) is established and it can be used for forecasting.

(2) The anticipated effect using the TDPFOGM $(1,1)$ model is better than the TDPGM model and the FOGM model under the same data condition.

\section{Data Availability}

The data used to support the findings of this study are included within the article.

\section{Conflicts of Interest}

The authors declare that they have no conflicts of interest.

\section{Acknowledgments}

The authors acknowledge the support of the applied fundamental research projects (major frontier projects) of Sichuan Province (no. 2016JC0314). 
TABle 2: The raw data of the example.

\begin{tabular}{lcccccccc}
\hline Year & 1 & 2 & 3 & 4 & 5 & 6 & 7 & 8 \\
Actual value & 0.16 & 1.11 & 1.92 & 2.24 & 3.03 & 3.3 & 4.16 & 4.64 \\
Year & 9 & 10 & 11 & 12 & 13 & 14 & 15 & 16 \\
Actual value & 5.18 & 5.6 & 6.25 & 6.39 & 7.35 & 8.18 & 8.57 \\
\hline
\end{tabular}

TABLE 3: Numerical results by the $\operatorname{TDPGM}(1,1), \operatorname{GM}(1,1), \operatorname{TDPGM}^{(0.25)}(1,1)$, and $\operatorname{GM}^{(0.25)}(1,1)$.

\begin{tabular}{|c|c|c|c|c|c|c|c|c|c|}
\hline Year & Natural gas consumption & $\operatorname{TDPGM}(1,1)$ & Eror(\%) & $\mathrm{GM}(1,1)$ & Eror(\%) & $\operatorname{TDPG}^{(0.25)}(1,1)$ & Eror(\%) & $\mathrm{GM}^{(0.25)}(1,1)$ & Eror(\%) \\
\hline 1995 & 177.53 & 177.53 & 0 & 177.53 & 0 & 177.53 & 0 & 177.53 & 0 \\
\hline 1996 & 188.05 & 180.88 & 3.81 & 343.87 & 82.86 & 186.45 & 0.85 & 185.64 & 1.28 \\
\hline 1997 & 176.13 & 193.11 & 9.46 & 184.26 & 4.62 & 190.65 & 8.24 & 189.35 & 7.51 \\
\hline 1998 & 218.7 & 206.39 & 5.63 & 204.11 & 6.67 & 215.94 & 1.26 & 207.48 & 5.13 \\
\hline 1999 & 215.34 & 222.06 & 3.12 & 226.11 & 5.00 & 223.14 & 3.62 & 221.26 & 2.75 \\
\hline 2000 & 245.03 & 241.61 & 1.40 & 250.47 & 2.22 & 235.75 & 3.79 & 240.34 & 1.91 \\
\hline 2001 & 274.3 & 266.66 & 2.79 & 277.45 & 1.15 & 276.62 & 0.85 & 268.57 & 2.09 \\
\hline 2002 & 291.84 & 299.00 & 2.45 & 307.34 & 5.31 & 289.28 & 0.88 & 305.29 & 4.61 \\
\hline 2003 & 339.08 & 340.60 & 0.45 & 340.46 & 0.41 & 326.92 & 3.59 & 347.21 & 2.40 \\
\hline 2004 & 396.72 & 393.61 & 0.79 & 377.14 & 4.94 & 391.64 & 1.28 & 384.94 & 2.97 \\
\hline MAPE (\%) & & & 3.01 & & 11.32 & & 2.44 & & 3.06 \\
\hline 2005 & 467.63 & 460.40 & 1.55 & 417.77 & 10.66 & 451.32 & 3.49 & 448.38 & 4.12 \\
\hline 2006 & 561.41 & 543.58 & 3.18 & 462.78 & 17.57 & 543.84 & 3.13 & 533.06 & 5.05 \\
\hline 2007 & 705.23 & 646.00 & 8.40 & 512.64 & 27.31 & 658.56 & 6.62 & 624.31 & 11.47 \\
\hline 2008 & 812.94 & 770.82 & 5.18 & 567.87 & 30.15 & 771.85 & 5.05 & 751.51 & 7.56 \\
\hline 2009 & 895.2 & 921.47 & 2.93 & 629.05 & 29.73 & 876.45 & 2.09 & 867.73 & 3.07 \\
\hline 2010 & 1069.41 & 1101.76 & 3.03 & 696.83 & 34.84 & 1020.62 & 4.56 & 1016.15 & 4.98 \\
\hline 2011 & 1305.3 & 1315.85 & 0.81 & 771.90 & 40.86 & 1203.47 & 7.80 & 1215.32 & 6.89 \\
\hline 2012 & 1463 & 1568.31 & 7.20 & 855.07 & 41.55 & 1412.62 & 3.44 & 1406.28 & 3.88 \\
\hline 2013 & 1705.37 & 1864.18 & 9.31 & 947.19 & 44.46 & 1685.16 & 1.19 & 1675.61 & 1.75 \\
\hline MAPE (\%) & & & 4.62 & & 30.79 & & 4.15 & & 5.42 \\
\hline
\end{tabular}

TABLE 4: Numerical results by the $\operatorname{GM}(1,1), \operatorname{GM}^{(0.5)}(1,1)$, and $\operatorname{TDPGM}^{(0.5)}(1,1)$.

\begin{tabular}{|c|c|c|c|c|c|c|c|}
\hline Year & Actual value & $\mathrm{GM}(1,1)$ & Error (\%) & $\mathrm{GM}^{(0.5)}(1,1)$ & Error $(\%)$ & $\operatorname{TDPGM}^{(0.5)}(1,1)$ & Error (\%) \\
\hline 1 & 0.155 & 0.155 & 0.00 & 0.155 & 0.00 & 0.155 & 0.00 \\
\hline 2 & 1.11 & 2.29 & 106.31 & 1.11 & 0.00 & 1.11 & 0.00 \\
\hline 3 & 1.92 & 2.54 & 32.29 & 1.86 & 3.12 & 1.87 & 2.60 \\
\hline 4 & 2.24 & 2.83 & 26.34 & 2.44 & 8.93 & 2.43 & 8.48 \\
\hline 5 & 3.03 & 3.15 & 3.96 & 2.97 & 1.98 & 2.99 & 1.32 \\
\hline 6 & 3.30 & 3.50 & 6.06 & 3.49 & 5.76 & 3.45 & 4.55 \\
\hline 7 & 4.16 & 3.89 & 6.49 & 3.99 & 4.09 & 4.10 & 1.44 \\
\hline 8 & 4.64 & 4.33 & 6.68 & 4.51 & 2.80 & 4.55 & 1.94 \\
\hline 9 & 5.18 & 4.81 & 7.14 & 5.04 & 2.70 & 5.11 & 1.35 \\
\hline 10 & 5.60 & 5.35 & 4.46 & 5.59 & 0.18 & 5.59 & 0.18 \\
\hline 11 & 6.25 & 5.95 & 4.80 & 6.15 & 1.60 & 6.21 & 0.64 \\
\hline 12 & 6.39 & 6.62 & 3.60 & 6.73 & 5.32 & 6.41 & 0.31 \\
\hline 13 & 7.35 & 7.36 & 0.14 & 7.35 & 0.00 & 7.34 & 0.14 \\
\hline 14 & 8.18 & 8.19 & 0.12 & 7.99 & 2.32 & 8.61 & 0.24 \\
\hline 15 & 8.57 & 9.10 & 6.18 & 8.66 & 1.05 & 8.61 & 0.47 \\
\hline 16 & 9.27 & 10.12 & 9.17 & 9.34 & 0.76 & 9.29 & 0.22 \\
\hline MAPE (\%) & & 14.07 & & 2.53 & & 1.49 & \\
\hline
\end{tabular}




\section{References}

[1] Q. Wang, X. Chen, A. N. Jha, and H. Rogers, "Natural gas from shale formation - the evolution, evidences and challenges of shale gas revolution in United States," Renewable \& Sustainable Energy Reviews, vol. 30, pp. 1-28, 2014.

[2] D. Spence, “The Shale Gas Revolution Continues," Power, vol. 157, no. 2, 2013.

[3] Y. Chang, X. Liu, and P. Christie, "Emerging shale gas revolution in China," Environmental Science \& Technology, vol. 46, no. 22, pp. 12281-12282, 2012.

[4] D. Hu and S. Xu, "Opportunity, challenges and policy choices for China on the development of shale gas," Energy Policy, vol. 60, pp. 21-26, 2013.

[5] Z. Wang, "The Influence of Shale Gas Revolution upon Geopolitics and Economy," in LISS 2013, pp. 711-716, Springer, 2015.

[6] J. L. Deng, "Introduction to grey system theory," The Journal of Grey System, vol. 1, no. 1, pp. 1-24, 1989.

[7] S. J. Wang, W. L. Wang, and C. T. Huang, "Improving inventory effectiveness in RFIF-enabled global supply chain with Grey forecasting model," The Journal of Strategic Information Systems, vol. 20, pp. 307-322, 2011.

[8] X. An, D. Jiang, M. Zhao, and C. Liu, "Short-term prediction of wind power using EMD and chaotic theory," Communications in Nonlinear Science and Numerical Simulation, vol. 17, no. 2, pp. 1036-1042, 2012.

[9] K. W. Hipel, “Grey Systems," Grey Systems: Theory and Applications, vol. 1, no. 3, pp. 274-275, 2011.

[10] C.-T. Lin and S.-Y. Yang, "Forecast of the output value of Taiwan's opto-electronics industry using the Grey forecasting model," Technological Forecasting \& Social Change, vol. 70, no. 2, pp. 177-186, 2003.

[11] C.-S. Lin, F.-M. Liou, and C.-P. Huang, "Grey forecasting model for $\mathrm{CO}_{2}$ emissions: a Taiwan study," Applied Energy, vol. 88, no. 11, pp. 3816-3820, 2011.

[12] E. Kayacan and O. Kaynak, "Single-step ahead prediction based on the principle of concatenation using grey predictors," Expert Systems with Applications, vol. 38, no. 8, pp. 9499-9505, 2011.

[13] H. Liu and D.-L. Zhang, "Analysis and prediction of hazard risks caused by tropical cyclones in Southern China with fuzzy mathematical and grey models," Applied Mathematical Modelling: Simulation and Computation for Engineering and Environmental Systems, vol. 36, no. 2, pp. 626-637, 2012.

[14] X. Ma and Z. Liu, "Application of a novel time-delayed polynomial grey model to predict the natural gas consumption in China," Journal of Computational and Applied Mathematics, vol. 324, pp. 17-24, 2017.

[15] D. Akay and M. Atak, "Grey prediction with rolling mechanism for electricity demand forecasting of Turkey," Energy, vol. 32, no. 9, pp. 1670-1675, 2007.

[16] Y.-H. Wu and H. Shen, "Grey-related least squares support vector machine optimization model and its application in predicting natural gas consumption demand," Journal of Computational and Applied Mathematics, vol. 338, pp. 212-220, 2018.

[17] X. Ma, Y.-S. Hu, and Z.-B. Liu, "A novel kernel regularized nonhomogeneous grey model and its applications," Communications in Nonlinear Science and Numerical Simulation, vol. 48, pp. 51-62, 2017.

[18] U. Kumar and V. K. Jain, “Time series models (Grey-Markov, Grey Model with rolling mechanism and singular spectrum analysis) to forecast energy consumption in India," Energy, vol. 35, no. 4, pp. 1709-1716, 2010.
[19] X.-W. Ren, Y.-Q. Tang, J. Li, and Q. Yang, "A prediction method using grey model for cumulative plastic deformation under cyclic loads," Natural Hazards, vol. 64, no. 1, pp. 441-457, 2012.

[20] Y. Hu, E. MacKay, O. Ishkov, and A. Strachan, "Predicted and observed evolution of produced-brine compositions and implications for scale management," SPE Production and Operations, vol. 31, no. 3, pp. 270-279, 2016.

[21] X. Ma, Z. B. Liu, and Y. Wei, "A novel kernel regularized nonlinear GMC $(1, n)$ model and its application," Journal of Grey System, vol. 28, no. 3, pp. 97-109, 2016.

[22] H. Pao and C. Tsai, "Modeling and forecasting the $\mathrm{CO}_{2}$ emissions, energy consumption, and economic growth in Brazil," Energy, vol. 36, no. 5, pp. 2450-2458, 2011.

[23] J. Cui, S.-F. Liu, B. Zeng, and N.-M. Xie, "A novel grey forecasting model and its optimization," Applied Mathematical Modelling, vol. 37, no. 6, pp. 4399-4406, 2013.

[24] B. Ayvaz and A. O. Kusakci, "Electricity consumption forecasting for Turkey with nonhomogeneous discrete grey model," Energy Sources, Part B: Economics, Planning, and Policy, vol. 12, no. 3, pp. 260-267, 2017.

[25] N. Xie and A. D. Pearman, "Forecasting energy consumption in China following instigation of an energy-saving policy," Natural Hazards, vol. 74, no. 2, pp. 639-659, 2014.

[26] L. Wu, S. Liu, L. Yao, S. Yan, and D. Liu, "Grey system model with the fractional order accumulation," Communications in Nonlinear Science and Numerical Simulation, vol. 18, no. 7, pp. 1775-1785, 2013.

[27] C. Yuan, S. Liu, and Z. Fang, "Comparison of China's primary energy consumption forecasting by using ARIMA (the autoregressive integrated moving average) model and GM(1,1) model," Energy, vol. 100, pp. 384-390, 2016.

[28] L. Wu, S. Liu, H. Chen, and N. Zhang, "Using a novel grey system model to forecast natural gas consumption in China," Mathematical Problems in Engineering, vol. 2015, Article ID 686501, 7 pages, 2015.

[29] S. H. Mao, M. Y. Gao, X. P. Xiao, and M. Zhu, "A novel fractional grey system model and its application," Applied Mathematical Modelling, vol. 40, no. 7-8, pp. 5063-5076, 2016.

[30] Y. Yang and D. Xue, "Continuous fractional-order grey model and electricity prediction research based on the observation error feedback," Energy, vol. 115, pp. 722-733, 2016.

[31] W. Meng, D. Yang, and H. Huang, "Prediction of China's sulfur dioxide emissions by discrete grey model with fractional order generation operators," Complexity, vol. 2018, Article ID 8610679, 13 pages, 2018.

[32] Y. Yang and D. Xue, "An actual load forecasting methodology by interval grey modeling based on the fractional calculus," ISA Transactions $^{\circledR}$, pp. 1-10, 2017.

[33] L. Wu, X. Gao, Y. Xiao, Y. Yang, and X. Chen, "Using a novel multi-variable grey model to forecast the electricity consumption of Shandong Province in China," Energy, vol. 157, pp. 327-335, 2018 .

[34] S. Li, X. Ma, and C. Yang, "A novel structure-adaptive intelligent grey forecasting model with full-order time power terms and its application," Computers \& Industrial Engineering, vol. 120, pp. 53-67, 2018.

[35] Z.-X. Wang, Q. Li, and L.-L. Pei, "Grey forecasting method of quarterly hydropower production in China based on a data grouping approach," Applied Mathematical Modelling, vol. 51, pp. 302-316, 2017. 
[36] Y. G. Zhang, X. Yan, and Z. P. Wang, "GM(1,1) Grey prediction of Lorenz chaotic system," Chaos, Solitons \& Fractals, vol. 42, pp. 1003-1009, 2009. 


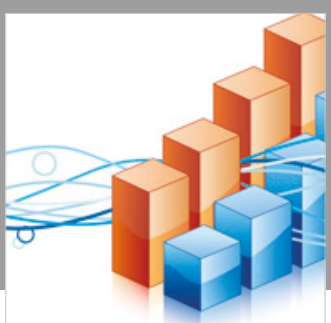

Advances in

Operations Research

\section{-n-m}
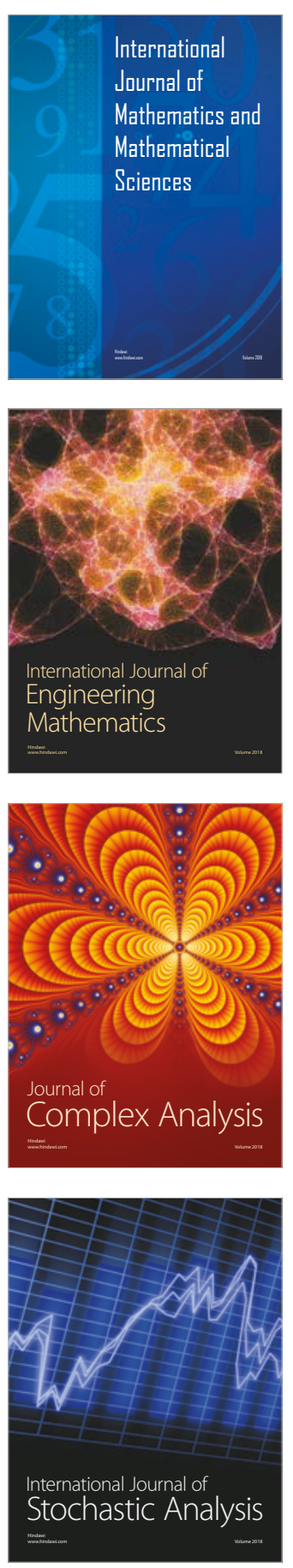
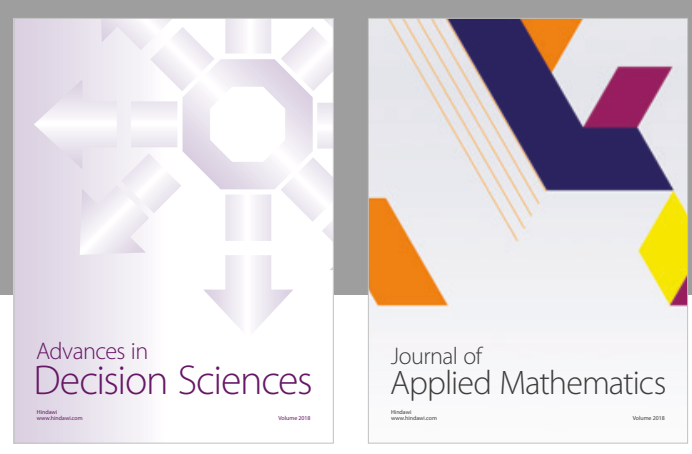

Journal of

Applied Mathematics
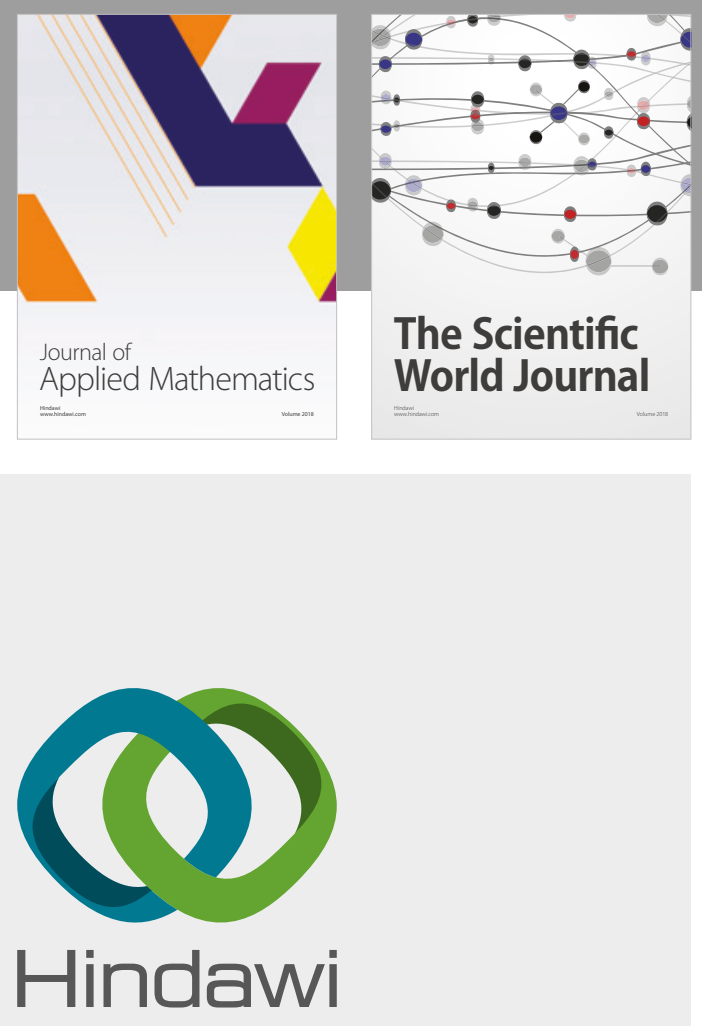

Submit your manuscripts at

www.hindawi.com

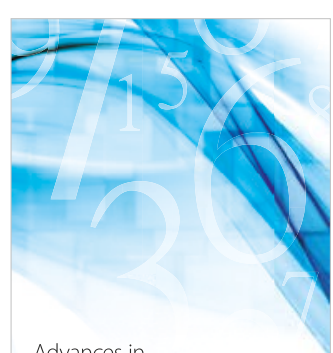

Advances in
Numerical Analysis
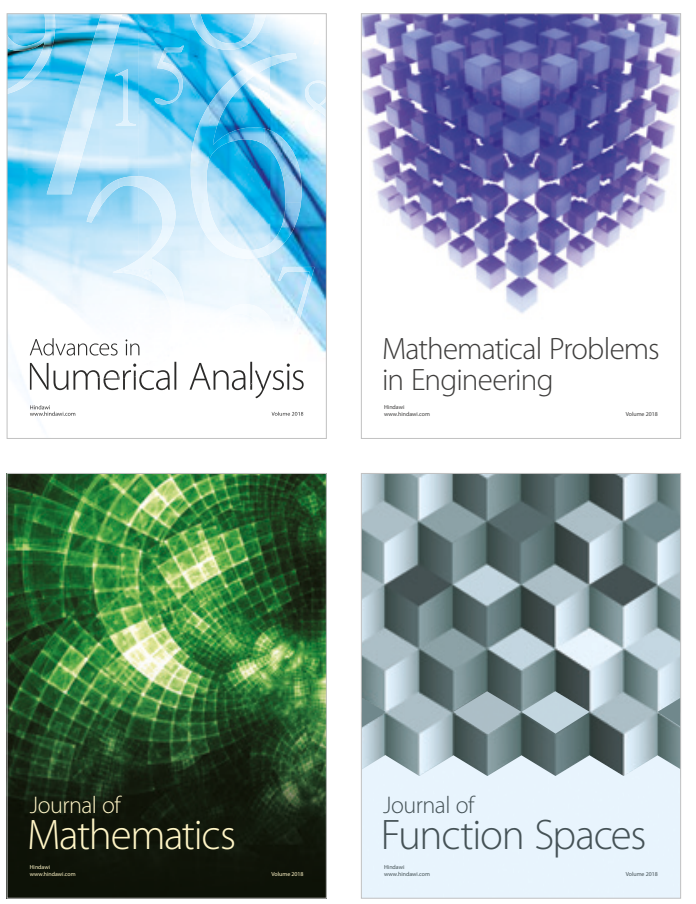

Mathematical Problems in Engineering

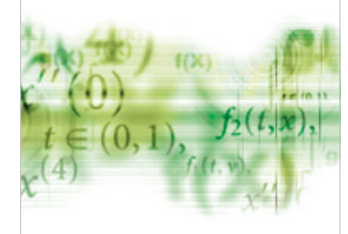

International Journal of

Differential Equations

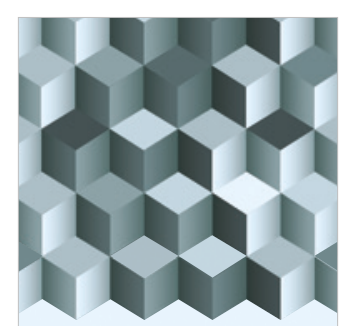

Journal of

Function Spaces

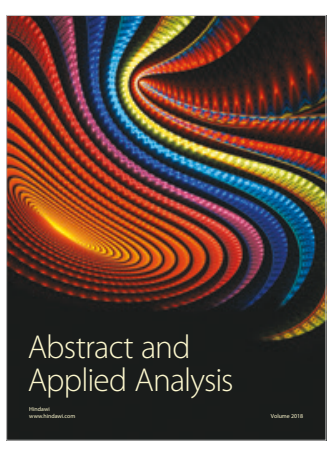

The Scientific

World Journal

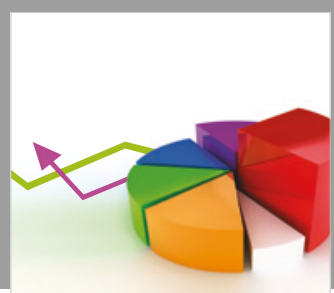

Journal of

Probability and Statistics
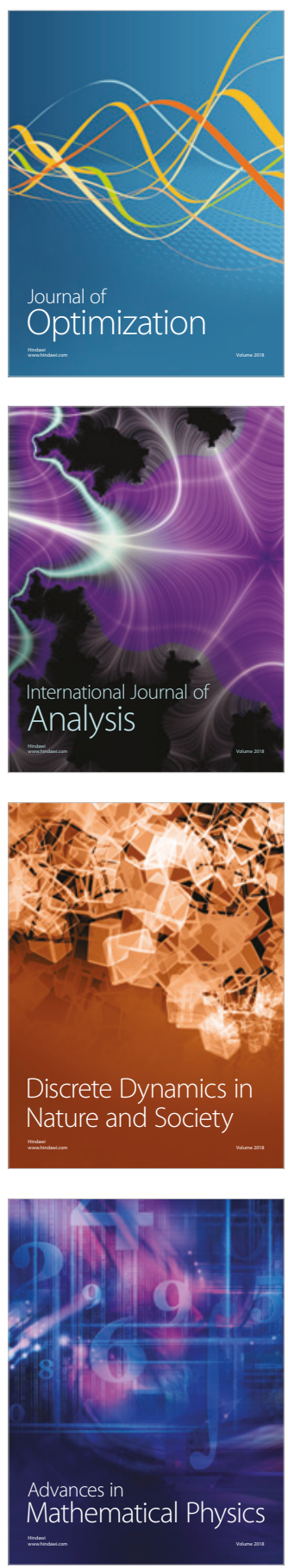